\title{
Insatisfação com a imagem corporal em professores de Educação Física atuantes na Educação Infantil
}

\section{Body image dissatisfaction in teachers of Physical Education acting in Chid Education}

\author{
Gaia Salvador Claumann, André de Araújo Pinto, Andreina Medeiros Costa, \\ Mateus Augusto Bim, Andreia Pelegrini
}

Como citar este artigo:

CLAUMANN, G. S.; PINTO, A. A.;

COSTA, A. M.; BIM, M. A.; PELECRINI,

A. Insatisfação com a imagem

corporal em professores de

Educação Física atuantes na

Educação Infantil. Revista Saúde

(Sta. Maria). 2019; 45 (2).

\begin{abstract}
Autor correspondente:
Nome: Andreia Pelegrini

E-mail: andreia.pelegrini@udesc.br

Telefone: +554836648695

Formação Profissional: Doutora

em Educação Física pela

Universidade Federal de Santa

Catarina (UFSC). Professora do programa de Pós-Graduação em

Ciências do Movimento Humano

da Universidade do Estado

de Santa Catarina (UDESC).

Florianópolis, Brasil.
\end{abstract}

Filiação Institucional: Universidade do Estado de Santa Catarina (UDESC)

Endereço para correspondência: Rua: Pascoal Simone n: 358

Bairro: Coqueiros

Cidade: Florianópolis

Estado: Santa Catarina

CEP: $88080-350$

Data de Submissão:

04/04/2019

Data de aceite:

04/08/2019

Conflito de Interesse: Não há conflito de interesse

\section{(cc) $\mathrm{BY}-\mathrm{NC}-\mathrm{ND}$}

\section{RESUMO}

Introdução: as condições de trabalho atuais podem ter inúmeras repercussões na saúde física e mental, destacando-se a imagem corporal de professores. Objetivo: analisar a insatisfação com a imagem corporal em professores de Educação Física atuantes na Educação Infantil. Método: participaram 38 professores (30 mulheres) da rede municipal de ensino de Florianópolis/SC, com média de idade de $35,7(9,0)$ anos. Foram coletadas informações demográficas, laborais, sobre imagem corporal (escala de silhuetas) e medidas antropométricas (massa corporal e estatura autorreferidas). Resultados: a insatisfação com a imagem corporal foi verificada em $68,4 \%$ dos professores. Aqueles que atuavam há dez anos ou mais no magistério $(p=0,040)$ e que trabalhavam até 40 horas por semana $(p=0,005)$ foram os mais insatisfeitos com a imagem corporal. Quanto ao status do peso, independentemente de apresentarem peso normal ou sobrepeso $(p=0,027)$, os professores estavam insatisfeitos. Conclusão: a maioria dos professores de Educação Física estava insatisfeita com sua imagem corporal, sendo que os mais insatisfeitos foram aqueles que atuavam na profissão há mais tempo, com maior carga horária semanal de trabalho $e$, independentemente, do status de peso.

PALAVRAS-CHAVE: Docentes; Educação infantil; Imagem corporal.

\section{ABSTRACT}

Introduction: the current working conditions can have numerous repercussions on physical and mental health, highlighting the body image of teachers. Goal: to analyze body image dissatisfaction in Physical Education teachers involved in Early Childhood Education. Method: participants were 38 teachers from the municial school system of Florianopolis/SC, with a mean age of 35.7 (9.0) years. Demographic, labor, body image (figure rating scale) and anthropometric measurements (self-reported body weight and height) were collected. Results: self-reported body weight and height was verified in $68.4 \%$ of the teachers. Those who worked ten years or more in teaching $(p=0.040)$ and who worked up to 40 hours per week $(p=0.005)$ were the most dissatisfied with body image. Regarding weight status, regardless of whether they were normal weight or overweight $(p=0.027)$, teachers were dissatisfied. Conclusion: the majority of physical education teachers were dissatisfied with their body image, and the most dissatisfied were those who had been in the profession for the longest time, with a higher weekly workload and regardless of their weight status.

KEYWORDS: Teachers; Child education; Body image. 


\section{INTRODUÇÃO}

O cenário atual do trabalho docente no Brasil é caracterizado por precarização e desvalorização social da profissão ${ }^{1,2}$. São inúmeros os fatores que contribuem para a formação desse quadro, destacando-se a baixa remuneração (principalmente se comparada à de outros profissionais com o mesmo nível de formação ou até mesmo inferior) ${ }^{3,4}$ e as más condições de trabalho ${ }^{2,3}$.

O sistema educacional, marcado pela intensificação do trabalho, sobrecarrega os professores com elevadas cargas horárias de trabalho (que incluem as atividades realizadas dentro e fora da sala de aula), podendo influenciar negativamente a saúde física e mental, além de mostrar a desvalorização do professor ${ }^{1,5}$.

Essa situação pode resultar em docentes cronicamente cansados, que apresentam padrão e qualidade de sono prejudicados, e pouco ou nenhum tempo para desfrutar de atividades de lazer e praticar hábitos saudáveis ${ }^{5,6}$. Tais fatores são preocupantes, especialmente quando considerados os professores de Educação Física, pois esses têm em seu corpo o instrumento de trabalho. Assim, a preocupação com o corpo se dá em relação ao seu desempenho, pois, o mesmo sofre com o desgaste decorrente da prática cotidiana, estando mais suscetíveis a lesões musculoesqueléticas em relação a professores de outras áreas, o que pode comprometer a demonstração de movimentos ${ }^{7}$.

Nesse contexto, estudos sobre a satisfação com a imagem corporal têm sido conduzidos em professores de modo geral ${ }^{8,10}$ e acadêmicos que estão cursando graduação em Educação Física ${ }^{11,14}$, porém, os estudos relacionados a imagem corporal de professores de Educação Física ainda são poucos na literatura, ${ }^{8,15}$. Dentre os resultados encontrados, observou-se que apenas $20,5 \%$ das professoras e $34 \%$ dos professores de escolas pública de Portugal mostraram-se satisfeitos com sua imagem corporal ${ }^{7}$, ademais, entre profissionais de Educação Física atuantes em academias, notouse que $40,1 \%$ deles apresentaram de leve a moderada distorção da imagem corporal e mostraram-se insatisfeitos com a mesma ${ }^{15}$.

Tais estudos são importantes, pois a forma física e a aparência do corpo se destacam devido às evidências de que os alunos consideram importante que o professor de Educação Física cuide do seu corpo, apresentando preferência por aqueles com corpos esteticamente bonitos ${ }^{15,17}$. Ainda, professores que têm maior satisfação com a imagem corporal tendem a ter motivação e autoestima melhores ${ }^{10}$, as quais podem ser valiosas na qualidade do papel desempenhado por eles no campo de trabalho.

Nesse sentido, torna-se necessário conhecer e compreender as relações dos professores de Educação Física com seu corpo e imagem corporal, principalmente por serem profissionais inseridos em um contexto no qual devem transmitir aos alunos conhecimentos sobre a educação e promoção da saúde, hábitos saudáveis e prevenção da obesidade ${ }^{18}$. Assim, é importante que apresentem conceitos sadios e positivos sobre seus corpos, pois ao falharem 
em cuidar da própria saúde podem ser menos eficazes em serem promotores na escola, sendo possível que criem preocupações alimentares e a respeito da imagem corporal entre estudantes mais vulneráveis, quando na verdade devem atuar para evitá-las e preveni-las ${ }^{18}$. Dessa forma, o presente estudo tem como objetivo analisar a insatisfação com a imagem corporal em professores de Educação Física atuantes na Educação Infantil.

\section{MATERIAIS E MÉTODOS}

Este estudo transversal faz parte do projeto "Qualidade de vida e nível de atividade física de lazer dos professores de Educação Física atuantes no ensino infantil das creches de Florianópolis - SC", aprovado pelo Comitê de Ética em Pesquisa com Seres Humanos da Universidade do Estado de Santa Catarina (parecer n0 275.116/2013).

A população do presente estudo foi constituída pelos professores de Educação Física, atuantes na educação infantil, em creches da rede municipal de ensino de Florianópolis - SC, em 2013. No município e no período em questão, foram identificadas 50 instituições de ensino com educação infantil, com as quais foi feito contato para apresentação do estudo e convite para a participação no mesmo. Em três creches não foi permitido o desenvolvimento da pesquisa, em quatro delas não foi possível contatar a coordenação, em uma não havia professor no cargo e em duas os professores estavam afastados. Desta forma, 40 professores de 40 creches foram convidados a fazer parte do estudo, dos quais 38 aceitaram responder ao questionário.

A coleta de dados ocorreu no ano de 2013, nas próprias creches, em horários disponibilizados pelos professores de Educação Física. Os docentes responderam a um questionário composto por questões demográficas (sexo, idade, cor da pele e estado civil), laborais, sobre imagem corporal e medidas antropométricas (massa corporal e estatura). Além disso, assinaram o Termo de Consentimento Livre e Esclarecido, aceitando participar voluntariamente da pesquisa. O pesquisador responsável esteve presente, junto ao professor de Educação Física, durante o preenchimento do questionário, para esclarecer possivveis dúvidas.

Os professores forneceram informações sobre seu grau de escolaridade (especialista, mestre ou doutor), carga horária semanal de trabalho como professor de Educação Física (categorizada em "até 30 horas semanais" e "até 40 horas semanais") e exercício de outra função remunerada. O tempo de atuação profissional no magistério foi mensurado por meio do ciclo de desenvolvimento profissional, adaptado por Nascimento e Graça ${ }^{19}$, o qual classifica a atuação em "entrada" (0-4 anos de docência), "consolidação" (5-9 anos), "diversificação" (10-19 anos) e "estabilização" (acima de 20 anos). Para fins de análise, os dados foram agrupados em duas categorias: "até nove anos" e "dez anos ou mais".

As medidas antropométricas de massa corporal e estatura foram autorreferidas pelos participantes, a partir das quais foi calculado o índice de massa corporal - IMC (massa corporal em kg dividida pela estatura, em metros, 
ao quadrado). O IMC foi utilizado para classificação do status do peso, conforme os pontos de corte recomendados para adultos pela Organização Mundial da Saúde ${ }^{20}\left(<18,5 \mathrm{~kg} / \mathrm{m}^{2}=\right.$ baixo peso; $18,50 \mathrm{~kg} / \mathrm{m}^{2}$ a $24,99 \mathrm{~kg} / \mathrm{m}^{2}=$ peso normal; $25,00 \mathrm{~kg} / \mathrm{m}^{2}$ a $29,99 \mathrm{~kg} / \mathrm{m}^{2}=$ sobrepeso; e $\geq 30,00 \mathrm{~kg} / \mathrm{m}^{2}=$ obesidade). Nenhum professor apresentou baixo peso e apenas um apresentou obesidade, o qual foi agrupado à categoria "sobrepeso".

A insatisfação com a imagem corporal foi verificada por meio da escala de silhuetas corporais de Stunkard, Sorensen e Schulsinge ${ }^{21}$, validada para adultos brasileiros por Scagliusi et al. ${ }^{22}$. O instrumento consiste em nove figuras de silhuetas corporais, de ambos os sexos, sendo que a figura um representa a magreza extrema e a nove, obesidade. Os professores foram instruídos a indicar qual das figuras consideravam melhor representar sua forma física atual (silhueta real) e com qual delas gostariam de se parecer, aproximadamente (silhueta ideal). 0 valor da silhueta real foi subtraído da silhueta ideal e quando o resultado foi igual a zero os participantes foram considerados satisfeitos, quando maior do que zero insatisfeitos pelo excesso, e, quando menor, insatisfeitos pela magreza.

Quanto às análises estatísticas, foram conduzidas análises descritivas (média, desvio padrão e distribuição de frequências) para caracterização dos participantes. Os testes Exato de Fisher ou Qui-quadrado foram utilizados para investigar possíveis associações entre a satisfação com a imagem corporal, as variáveis demográficas, laborais e 0 status de peso. $O$ nível de significância adotado em todas as análises foi de $p<0,05$ e as mesmas foram realizadas no software IBM SPSS Statistics, 20.0.

\section{RESULTADOS}

Participaram do estudo 30 mulheres e oito homens, com média de idade de 35,7 $(9,0)$ anos. Conforme apresentado na Tabela 1, a maioria dos professores de Educação Física atuantes na Educação Infantil do município de Florianópolis tinham idade inferior a 35 anos, eram de cor da pele branca, tinham companheiro e apresentavam peso normal. Quanto às características laborais, maior parte deles possuía especialização, atuava profissionalmente há dez anos ou mais e trabalhava até 40 horas por semana.

Tabela 1. Características gerais dos professores de Educação Física atuantes na Educação Infantil do município de Florianópolis/SC. (2013).

\begin{tabular}{lr}
\hline Variáveis & $\mathbf{n}(\%)$ \\
\hline Sexo & \\
Masculino & $8(21,1)$ \\
Feminino & $30(78,9)$ \\
\hline
\end{tabular}




\section{Faixa etária}

$<35$ anos

$20(52,6)$

$\geq 35$ anos

$18(47,4)$

\section{Cor da pele}

Branca

Preta/parda/amarela/indígena

$6(15,8)$

\section{Estado civil}

Com companheiro

$25(65,8)$

Sem companheiro

$13(34,2)$

\section{Grau de escolaridade}

Especialista

Mestrado/Doutorado $7(18,4)$

\section{Tempo de atuação}

Até nove anos

$22(57,9)$

Dez anos ou mais

$16(42,1)$

\section{Carga horária semanal}

Até 30 horas

$10(26,3)$

Até 40 horas $28(73,7)$

\section{Status do peso}

Peso normal

Sobrepeso $14(36,8)$

n: frequência absoluta; \%: frequência relativa.

Na Figura 1 são apresentadas as prevalências de professores satisfeitos e insatisfeitos com sua imagem corporal. Foi verificado que a maioria deles apresentou insatisfação com a imagem corporal (68,4\%), sendo superior a insatisfação pelo excesso $(65,8 \%)$.

Figura 1. Insatisfação com a imagem corporal em professores de Educação Física atuantes na Educação Infantil do município de Florianópolis/SC. (2013).
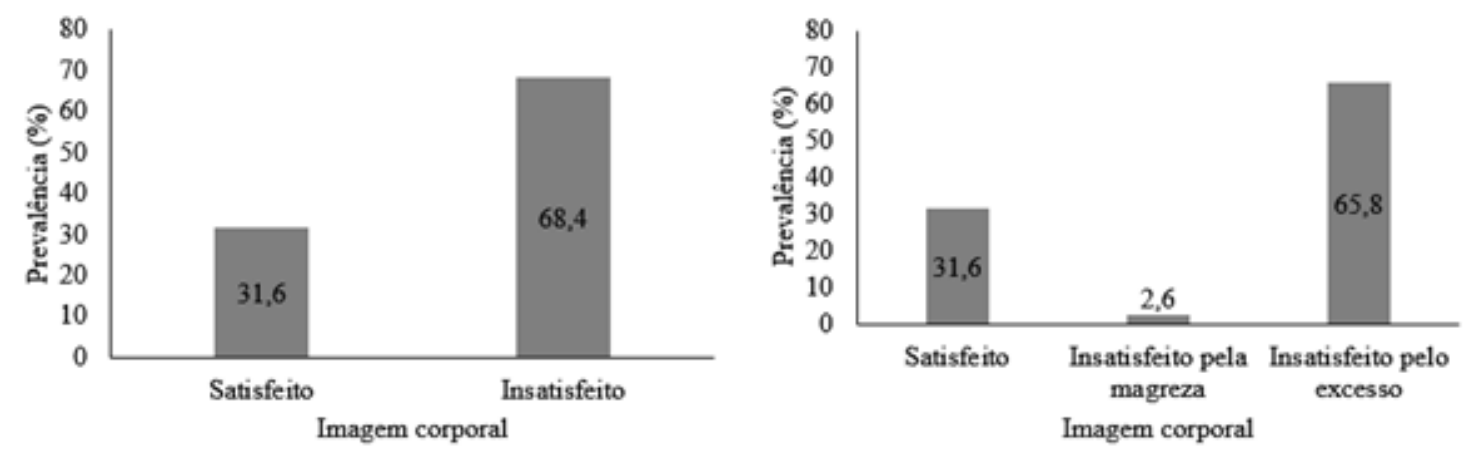
A Tabela 2 apresenta a associação da imagem corporal com as variáveis demográficas, laborais e status de peso de professores de Educação Física. Verificou-se associação da imagem corporal com as variáveis tempo de atuação profissional, carga horária semanal e status do peso. Observou-se que os professores que atuavam há dez anos ou mais no magistério e que trabalhavam até 40 horas por semana, apresentavam-se mais insatisfeitos com a imagem corporal (ajuste residual $\geq 2$ 2). Em relação ao status do peso, observou-se associação significativa, porém, independentemente do IMC (peso normal, sobrepeso), há presença de insatisfação com a imagem corporal (ajuste residual $\geq 2$ ).

Tabela 2. Associação entre imagem corporal, variáveis demográficas, laborais e status do peso em professores de Educação Física atuantes na Educação Infantil do município de Florianópolis/SC (2013).

\begin{tabular}{|c|c|c|c|}
\hline \multirow[t]{2}{*}{ Variáveis } & Satisfeitos & Insatisfeitos & \multirow[t]{2}{*}{$p$-valor } \\
\hline & $\mathrm{n}(\%)$ & $\mathrm{n}(\%)$ & \\
\hline Sexo & & & 0,232 \\
\hline Masculino & $4(33,3)$ & $4(15,4)$ & \\
\hline Feminino & $8(66,7)$ & $22(84,6)$ & \\
\hline Faixa etária & & & 0,086 \\
\hline$<35$ anos & $9(75,0)$ & $11(42,3)$ & \\
\hline$\geq 35$ anos & $3(25,0)$ & $15(57,7)$ & \\
\hline Estado civil & & & $0,163^{*}$ \\
\hline Com companheiro & $6(50,0)$ & $19(73,1)$ & \\
\hline Sem companheiro & $6(50,0)$ & $7(26,9)$ & \\
\hline Cor da pele & & & 0,357 \\
\hline Branca & $9(75,0)$ & $23(88,5)$ & \\
\hline Preta/parda/amarela/indígena & $3(25,0)$ & $3(11,5)$ & \\
\hline Grau de escolaridade & & & 1,000 \\
\hline Especialista & $10(83,3)$ & $21(80,8)$ & \\
\hline Mestrado/Doutorado & $2(16,7)$ & $5(19,2)$ & \\
\hline Tempo de atuação & & & 0,040 \\
\hline Até 9 anos & $10(83,3)$ & $12(46,2)$ & \\
\hline 10 anos ou mais & $2(16,7)$ & $14(53,8)$ & \\
\hline Carga horária semanal & & & 0,005 \\
\hline Até 30 horas & $7(58,3)$ & $3(11,5)$ & \\
\hline Até 40 horas & $5(41,7)$ & $23(88,5)$ & \\
\hline Status do peso & & & 0,027 \\
\hline Peso normal & $11(91,7)$ & $13(50,0)$ & \\
\hline Sobrepeso & $1(8,3)$ & $13(50,0)$ & \\
\hline
\end{tabular}

n: frequência absoluta; \%: frequência relativa.

${ }^{*}$ Qui-quadrado 


\section{DISCUSSÃO}

Os resultados do presente estudo revelaram que a maioria dos professores de Educação Física atuantes na Educação Infantil do município de Florianópolis/SC estava insatisfeita com sua imagem corporal, com proporção maior de insatisfeitos pelo excesso. Verificou-se associação da imagem corporal com o tempo de atuação profissional, carga horária de trabalho semanal e status do peso.

A prevalência geral de insatisfação com a imagem corporal observada nos professores de Educação Física se assemelha aos estudos conduzidos em universitários desse curso, os quais apontam que entre $60 \%$ e $80 \%$ dessa população está insatisfeita ${ }^{11,14}$, independentemente do tipo de habilitação (Licenciatura ou Bacharelado) e da fase que estavam cursando. Neste sentido, é possível supor que os professores do presente estudo apresentavam insatisfação anterior à sua atuação profissional, pois, conforme sugerem Yager e O'Dea ${ }^{18}$, indivíduos previamente insatisfeitos com seu corpo e aparência, ou que sofrem com distúrbios alimentares, são atraídos para áreas da saúde como a Educação Física e a Nutrição, por exemplo, a fim de compreender e solucionar os problemas e as condições em que se encontram.

Dessa forma, não se deve ignorar as dificuldades encontradas por esses profissionais em sua rotina de trabalho, especialmente em escolas públicas, pois o grande volume de trabalho que assumem faz com que, muitas vezes, seu potencial papel de tutor de resiliência fique esquecido ou em segundo plano ${ }^{23}$, fazendo com que ele não tenha tempo para se dedicar a si deixando de lado o autocuidado com a saúde do corpo de uma forma geral.

Em relação aos fatores associados à insatisfação com a imagem corporal, observou-se que os professores com maior tempo de atuação (dez anos ou mais) foram os mais insatisfeitos. Acredita-se, portanto, que além de possível insatisfação prévia, a prática profissional e as exigências relacionadas ao corpo, inerentes à mesma, possam contribuir para o agravamento de sentimentos negativos sobre a imagem corporal. Apontando para a mesma direção, Lemos, Nascimento e Borgatto ${ }^{24}$ verificaram que professores de Educação Física em início de carreira percebiam seu estilo de vida mais positivamente comparados àqueles com carreiras mais avançadas. Apontando para a mesma direção, Both et al. ${ }^{6}$ verificaram que professores de Educação Física em início de carreira apresentavam menor nível de insatisfação no trabalho e carreira quando comparados àqueles com carreiras mais avançadas. Ou seja, quanto maior o tempo de atuação, a percepção do estilo de vida, de modo geral, era pior. Entende-se que essa interpretação pode ser extrapolada à imagem corporal.

A permanência do professor na escola, de forma geral, parece ter impacto em sua imagem corporal. Assim como o tempo de atuação profissional, a carga horária semanal de trabalho também se associou ao desfecho. A prevalência de insatisfação com a imagem corporal foi superior entre os professores que trabalhavam até 40 horas semanais em relação àqueles que trabalhavam até 30 horas. Ressalta-se ainda que $39,3 \%$ dos professores com carga horária de 
trabalho de até 40 horas possuem alguma outra função remunerada (dados não apresentados), além da sua atuação nas creches. Essa condição pode estar relacionada à necessidade de complementar a renda, visto que frequentemente a baixa remuneração é apontada como fator de preocupação pelos professores de Educação Física²5,26.

Nesse sentido, o acúmulo da quantidade de horas trabalhadas e/ou de trabalho realizado pode sobrecarregar os professores tanto física quanto psicologicamente, tendo impacto direto em sua imagem corporal, assim como no bemestar e saúde em geral. Dessa forma, a redução da carga horária de trabalho e/ou uma melhor remuneração parecem ser medidas necessárias e que precisam ser consideradas pelas autoridades responsáveis. Isto porque, possuindo menor carga de trabalho é maior a possibilidade desses professores conseguirem distribuir e aproveitar melhor o tempo dedicado ao trabalho, família e lazer ${ }^{24}$. Da mesma forma, recebendo melhores salários, os docentes não precisariam buscar outras atividades laborais ${ }^{26}$, para suprir sua condição financeira e, assim apresentariam uma quantidade menor de tarefas extras a cumprir, podendo dedicar-se às questões de interesse individual, incluindo seu corpo.

No tocante, Castelo-Branco e Pereira ${ }^{10}$ apontam que a satisfação pessoal e profissional dos professores é resultante de uma interação entre o trabalho e o lazer, no qual a conciliação desses aspectos possa ser conduzida para um estilo de vida mais saudável, fazendo com que a ocupação de tempos livres a partir de centros de reflexão e lazer tragam ainda mais satisfação para os docentes. Os autores ainda destacam que é importante a promoção da saúde mental e física para seu próprio bem-estar e, consequentemente, auxiliarão na transmissão de tais comportamentos para os alunos.

Ademais, houve associação do status do peso com a insatisfação com a imagem corporal. A respeito da relação entre esses fatores, a literatura é consistente em apontar que indivíduos adultos com excesso de peso são os mais insatisfeitos e isso também é observado em acadêmicos de Educação Física ${ }^{12,13,27,28}$. Entretanto, no presente estudo, os professores apresentaram insatisfação independentemente de terem peso normal ou excesso de peso. Isso pode indicar que a insatisfação com o corpo e, acredita-se, as preocupações com a aparência e forma física, sejam condições fortemente relacionadas à profissão, ou até mesmo inerentes à mesma. Senão, outros fatores também relacionados à prática, investigados no presente estudo, parecem ter maior impacto na imagem corporal do que o status do peso.

Dentre as principais limitações deste estudo, destaca-se o uso de medidas de massa corporal e estatura autorreferidas, contudo, esse método de obtenção das variáveis em questão é considerado válido para indivíduos adultos $^{29}$. Além disso, a amostra alcançada pode ser considerada pequena $(n=38)$, porém, é representativa da população de professores de Educação Física da educação infantil da rede municipal de Florianópolis, visto que na época de realização das coletas de dados havia 47 docentes atuando nas 50 creches elegíveis à participação no estudo.

Ressalta-se que, de acordo com a literatura consultada, este é um dos primeiros estudos no Brasil a investigar aspectos da imagem corporal na população de professores de Educação Física da educação infantil. Os resultados 
encontrados alertam para a necessidade de investigações que sejam conduzidas com um maior número de participantes, de outras regiões do país bem como com professores dos outros anos de ensino, considerando que se trata de um aspecto intimamente relacionado à saúde e ao bom desempenho da profissão desses indivíduos.

É por meio dos professores de Educação Física que a escola tem possibilidade de proporcionar apoio aos alunos que possuem alguma condição irregular de saúde, para que recebam a devida atenção e aconselhamento adequado ${ }^{30}$. Assim, é imprescindível que esses profissionais sejam capazes e estejam aptos a transmitir valores de saúde, higiene e estética de maneira eficaz e, para que isso seja possível, é importante que esses aspectos estejam incorporados em suas vidas pessoais.

\section{CONSIDERAÇÕES FINAIS}

A insatisfação com a imagem corporal foi observada na maioria dos professores de Educação Física atuantes na educação infantil do município de Florianópolis/SC. Professores que atuavam na carreira há mais tempo e que trabalhavam mais horas por semana foram os mais insatisfeitos com sua imagem corporal, bem como aqueles que se encontravam com peso normal ou acima dele.

\section{REFERÊNCIAS}

1. Da Silva LMS, Pereira FD, Novello TP. Silveira DS. Relação entre a desvalorização profissional e o mal-estar docente. RELACult-Revista Latino-Americana de Estudos em Cultura e Sociedade. 2018 fev 4: 1-10.

2. Meira TRM, Cardoso JF, Vilela ABA, Amorim CR, Rocha SV, Andrade NA, et al. Percepções de professores sobre trabalho docente e repercussões sobre sua saúde. Revista Brasileira em Promoção da Saúde. 2014 abr./jun; 27(2): 276-282.

3. Cassettari N, Scaldelai VF, Frutuoso PC. Exoneração a pedido de professores: estudo em duas redes municipais paulistas. Educação e Sociedade. 2014 jul.-set; 35(128): 909-27.

4. Barbosa A. Salários docentes, financiamento e qualidade da educação no Brasil. Educação e Realidade. 2014 abr.jun; 39(2): 511-532. 
5. Assunção AA, Oliveira DA. Intensificação do trabalho e saúde dos professores. Educação e Sociedade. 2009 maio/ago; 30(107): 349-372.

6. Both J, Nascimento JV, Sonoo CN, Lemos CAF, Borgatto AF. Bem estar do trabalhador docente de educação física da região sul do Brasil de acordo com os ciclos vitais. Revista Brasileira de Educação Física e Esporte. 2014 jan.-mar; 28(1): 77-93.

7. Gooseens L, Vercruysse S, Cardon G, Haerens L, Wityrouw E, De Clercq D. Musculoskeletal injuries in physical education versus non-physical education teachers: a prospective study. Journal of Sports Sciences. 2016; 34(12): 1107-1115.

8. Cruz J, Matos MG, Marques A, Diniz JA. Como percecionam os professores a sua imagem corporal. Boletim Sociedade Portuguesa de Educação Física. 2018; (41), 27-39.

9. Fantin M. Conhecimento estético, tecnologia da sensibilidade e experiências formativas de crianças, jovens e professores. Revista tempos e espaços em educação. 2018 jul./set; 11(26): 39-45.

10. Castelo-Branco MCASH, Pereira AMS. A auto-estima, a satisfação com a imagem corporal e o bem-estar docente. Psicologia, Educação e Cultura. 2001; (2) :335-245.

11. Ferrari EP, Silva DAS, Petroski EL. Associação entre percepção da imagem corporal e estágios de mudança de comportamento em acadêmicos de educação física. Revista Brasileira de Cineantropometria e Desempenho Humano. 2012 jul; 14(5): 535-544.

12. Claumann GS, Pareira EF, Inácio S, Santos MC, Martins AC, Pelegrini A. Satisfação com a imagem corporal em acadêmicos ingressantes em cursos de educação física. Revista da Educação Física/UEM. 2014 trim.; 25(4): $575-583$.

13. Frank R, Claumann GS, Pinto AA, Cordeiro PC, Felden EPG, Pelegrini, A. Fatores associados à insatisfação com a imagem corporal em acadêmicos de Educação Física. Jornal Brasileiro de Psiquiatria. 2016 março; 65(2): 161-7. 
14. Legey S, Lamego MK, Lattari E, Campos C, Paes F, Sancassiani F, et al. Relationship Among Body Image, Anthropometric Parameters and Mental Health in Physical Education Students. Clinical Practice and Epidemiology in Mental Health. 2016; 12: 177-187.

15. Vilhena LM, Santos TM, Palma A, Murão L. Avaliação da imagem corporal em professores de educação física atuantes no fitness na cidade do Rio de Janeiro. Rev. Bras. Ciênc. Esporte. 2012 abr./jun; 34(2): 449-464.

16. Lüdorf SMA. The body and training for physical education teachers. Interface Comunic., Saúde, Educ. 2009 jan./mar; 13(28): 99-110.

17. Rosa JTV, Assis MR. A expectativa dos frequentadores de academia em relação ao corpo do professor de educação física. Corpus et Scientia. 2013 jan; 9(1) : 79-88.

18. Yager Z, O'dea J. Body image, dieting and disordered eating and activity practices among teacher trainees: implications for school-based health education and obesity prevention programs. Health Education Research, 2009 jun; 24 (3): 472-82.

19. Nascimento JV, Graça A. A evolução da percepção de competência profissional de professores de Educação Física ao longo da carreira docente. In: Congresso de Educação Física e Ciências do Desporto dos Países de Língua Portuguesa. 1998; 6 : 320-335.

20. World Health Organization. Obesity: preventing and managing the global epidemic: report of a WHO consultation on obesity. Geneva 1998.

21. Stunkard, AJ, Sorensen, T, Schulsinger, F. Use of the Danish Adoption Register for the study of obesity and thinness. Res Publ Assoc Res Nerv Ment Dis. 1983; 60: 115 120.

22. Scagliusi FB, Alvarenga M, Polacow VO, Coedás TA, Queiroz GKO, Coelho D, et.al. Concurrent and discriminant validity of the Stunkard's figure rating scale adapted into Portuguese. Appetite, 2006;47(1):77-82.

23. Neves AN, Hirata KM, Tavares MCGCF. Imagem corporal, trauma e resiliência: reflexões sobre o papel 
do professor de Educação Física. Psicologia Escolar e Educacional. 2015 janeiro/abril ;19(1):97-104.

24. Lemos CAF, Nascimento JV, Borgatto AF. Parâmetros individuais e sócio-ambientais da qualidade de vida percebida na carreira docente em Educação Física. Revista Brasileira de Educação Física e Esporte. 2007 abril/ jun;21(2):81-93.

25. Do Nascimento RK, Folle A, Rosa Al, Both J. Satisfação no trabalho dos professores de educação física da rede municipal de ensino de São José-SC. Journal of Physical Education. 2016; 27(1): 1-11.

26. Both J, Borgatto AF, Sonoo CN, Lemos CAF, Ciampolini C, Nascimento JV. Multiple Jobholding associated with the wellbeing of physical education teachers in southern Brazil. Educación Física y Deporte. 2016; 35(1):117140.

27. Alves FR, Souza EA, Paiva CS, Teixeira FAA. Insatisfação com a imagem corporal e fatores associados em universitários. Cinergis. 2017; 18(3): 204-209.

28. Pelegrini A, Sacomori C, Santos MC, Sperandio FS, Cardoso FL. Body image perception in women: prevalence and association with anthropometric indicators. Revista Brasileira de Cineantropometria e Desempenho Humano. 2014: 16(1): 58-65.

29. Coqueiro RS, Borges LJ, Araújo VC, Pelegrini A, Barbosa AR. Medidas auto-referidas são válidas para avaliação do estado nutricional na população brasileira? Revista Brasileira de Cineantropometria e Desempenho Humano. 2009; 11(1):113-119.

30. Knightsmith P, Sharpe H, Breen O, Treasure J, Schmidt U. 'My teacher saved my life' versus 'Teachers don't have a clue': an online survey of pupils' experiences of eating disorders. Child Adolesc Ment Health. 2014;19(2):131137. 